\title{
Quantitative evaluation of hemodynamic parameters by echocardiography in patients with postcardiotomy cardiac shock supported by extracorporeal membrane oxygenation
}

\author{
Fu-Yong Ye ${ }^{1}$, Yuwen Yang ${ }^{1}$, Xiaofang Li $^{2}$, Fei Lin ${ }^{2}$, Yin-Ting Liang ${ }^{2}$, and Jianhua Liu ${ }^{1}$ \\ ${ }^{1}$ The First Affiliated Hospital of Jinan University \\ ${ }^{2}$ Gaozhou People's Hospital
}

May 6, 2021

\begin{abstract}
Objective: To investigate the value of echocardiography in monitoring hemodynamics of postcardiotomy cardiac shock (PCS) patients before, during, and after weaning from extracorporeal membrane oxygenation (ECMO). Methods: Fifty-two patients were divided into a successful weaning group (Group A, n=23) and non-successful group (Group B, n=29). Hemodynamic parameters measured by echocardiography were collected before, during, and after ECMO. The intra-group changes and intergroup differences were analyzed. Results: In group A, the central venous pressure (CVP), proximal right ventricular outflow tract (RVOT), tricuspid annular plane systolic excursion (TAPSE), velocity of tricuspid valve (TVDV), and systolic velocity of tricuspid annulus ( $\left.\mathrm{s}^{6} \mathrm{TV}\right)$ during EMCO were significantly lower than before ECMO. After ECMO, left ventricular ejection fraction (LVEF), systolic velocity of mitral annulus $\left(\mathrm{s}^{6} \mathrm{MV}\right)$, and velocity-time integral of LV outflow tract (LVOT-VTI) were higher than pre-ECMO, and CVP, LVEF, s'MV, LVOT-VTI, RVOT, TAPSE, TVDV and s'TV were higher than during ECMO (all $\mathrm{p}<0.05$ ). In group B, compared to pre-ECMO, subjects exhibited decreased CVP, RVOT, TAPSE, TVDV and s'TV during ECMO. TAPSE, TVDV, and s'TV were continuously lower after ECMO, while CVP and RVOT became higher after ECMO (all $\mathrm{p}<0.05)$. After ECMO, LVEF, s'MV, LVOT-VTI, TAPSE, TVDV and s'TV in group A were higher than those in group $\mathrm{B}($ all $\mathrm{p}<0.05)$. Multiple logistic regression analysis showed that LVEF (OR=1.387, 95\%CI: 1.072-1.793, $\mathrm{p}=0.013$ ) and Tei index $(\mathrm{OR}=-0.005,95 \% \mathrm{CI}: 0.000-0.939, \mathrm{p}=0.047)$ were independent factors related to the successfulness of ECMO weaning. Conclusions: Quantitative assessment of both LV and RV by echocardiography is important for ECMO weaning.
\end{abstract}

\section{Hosted file}

Evaluation by TTE in patients with PCS supported by ECMO.pdf available at https://authorea. com/users/412324/articles/521047-quantitative-evaluation-of-hemodynamic-parametersby-echocardiography-in-patients-with-postcardiotomy-cardiac-shock-supported-byextracorporeal-membrane-oxygenation 

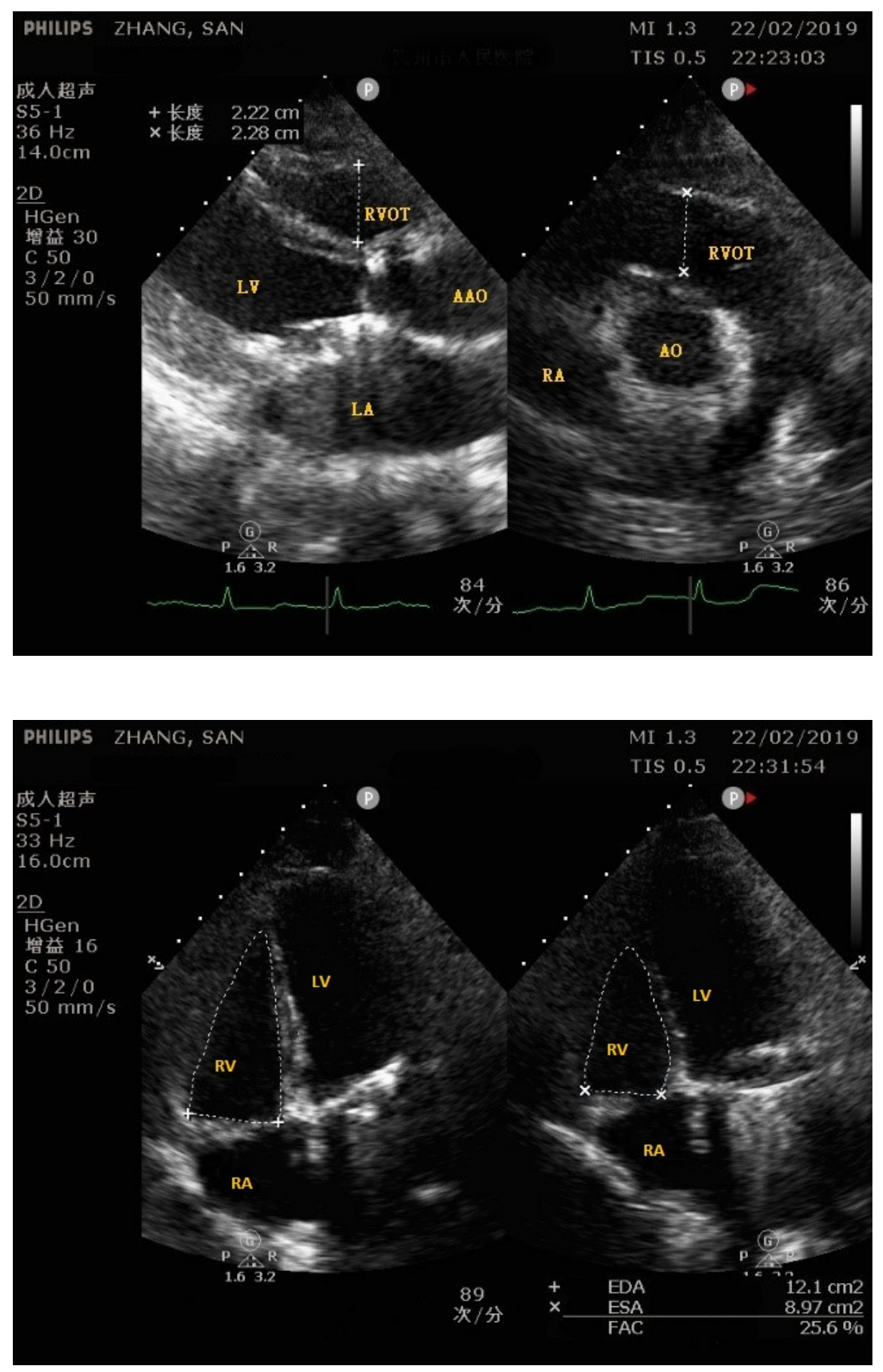

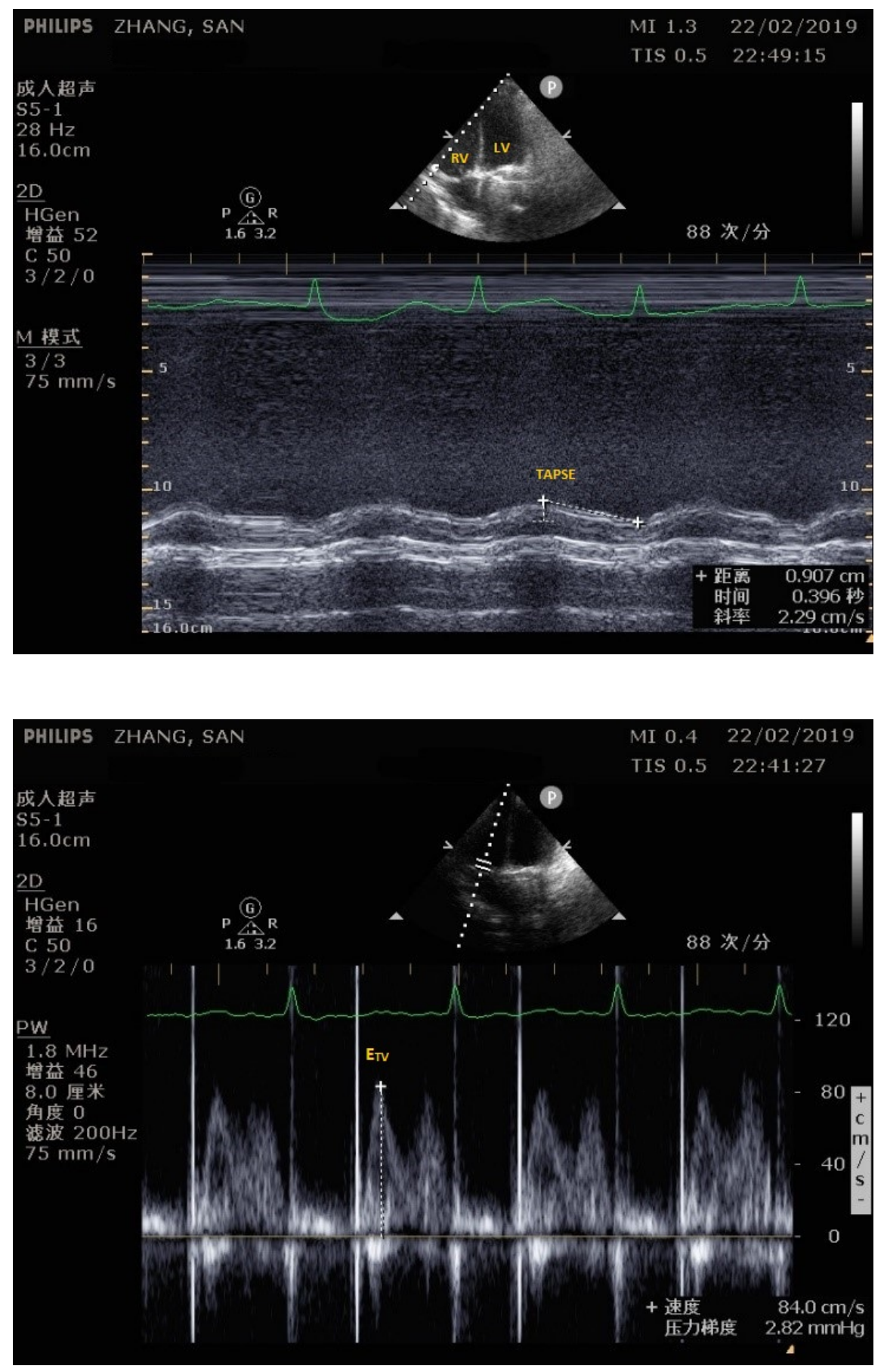


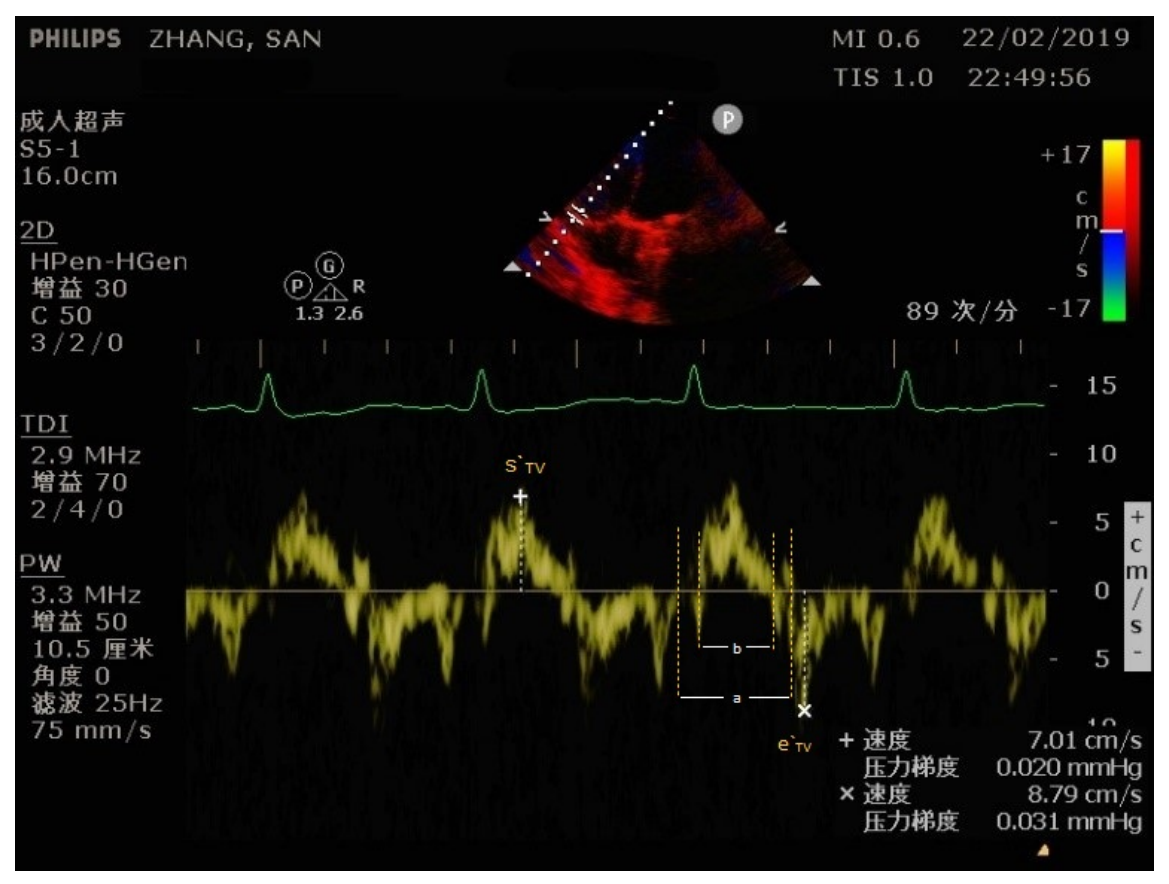

